\title{
A New, Easily Miniaturized Steerable Endoscope
}

\author{
BY PAUL BREEDVELD, \\ JULES S. SCHELTES, ESTHER M. BLOM, \\ AND JOHANNA E.I. VERHEIJ
}

Squid Tentacles

Provide Inspiration for the Endo-Periscope

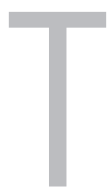

his article describes a new steerable endoscope for laparoscopic surgery. The steerable mechanism was inspired by the tentacles of squid and consists only of standard parts such as cables, coil springs, rings, and tubes. The mechanism can be easily miniaturized to a very small diameter, making it suitable for low-cost mass production of steerable endoscopes, instruments, and catheters. A patent for the mechanism has been applied for, and it is currently being commercialized.

Laparoscopic surgery is carried out using an endoscope and long and slender instruments that are inserted through small incisions in the abdominal wall (Figure 1). The endoscope contains a light source and a camera that displays a picture of the abdominal cavity on a monitor in the operation room. The surgeon uses the instruments to carry out the operation while estimating the spatial position of the instruments and the organs from the camera picture.

Technical advancements during the last 20 years have resulted in a reduction in the endoscope's diameter from $12 \mathrm{~mm}$ to $5 \mathrm{~mm}$ and in a strong improvement in image quality. A drawback of current endoscopes is that the movements of the camera are restricted. The incision acts like a fulcrum, limiting endoscope movements to four degrees of freedom (DOF) (Figure 2). As a result, it is impossible to observe organs from different sides and to look behind anatomic structures that obstruct the endoscope's field of view. Research on depth perception has shown that observing objects from different sides reduces the risk of mistakes when spatially interpreting a two-dimensional (2-D) image [1]-[4]. Being able to steer the camera around anatomic structures can be very useful for searching tumor metastases or performing surgery in cavities that are difficult to reach with conventional endoscopes.

To increase the maneuverability of the endoscopic camera, a new endoscope, the Endo-Periscope, has been developed at the Delft University of Technology in close cooperation with the Tokyo Institute of Technology. The Endo-Periscope (Figure 3) has a rigid shaft and a 2-DOF steerable tip with a miniature camera, enabling the surgeon to observe organs from the side and to look behind anatomic structures. The steerable tip is controlled via a spatial parallelogram-mechanism (Figure 4); the camera follows the handgrip movements exactly, and the handgrip is always parallel to the camera's line of sight. This provides intuitive control of the tip, showing how the camera is oriented in the abdominal cavity.

\section{Incompressible Steerable Spine Mechanisms}

The largest problem in the development of the Endo-Periscope was the construction of the steerable tip. The tip should be small, hollow, and able to bend in all 
directions over a large angle with a very small bending radius. The parallelogram-mechanism in Figure 4 consists of three rods (indicated by bold lines), which are connected to the handgrip and camera by means of universal joints (indicated by dots). If the mechanism is miniaturized to a real-size prototype, then the three universal joints in the tip must fit within a maximum diameter of $12 \mathrm{~mm}$ while leaving space for the cabling of the camera and the light source. The universal joints are placed beside each other, allowing each of them to be not more than approximately $2 \mathrm{~mm}$ in diameter. Since this leads to a very fragile construction, the parallelogram-mechanism was miniaturized in another way.

Steerable endoscopes are already used for inspecting hollow organs such as the stomach or colon. These gastroscopes or colonoscopes contain a long, flexible shaft and a 2-DOF steerable tip, which is controlled by means of control knobs. The tip contains a flexible construction of incompressible, ring-shaped elements, similar to the spine in a human body. In gastroscopes and colonoscopes, the spine is constructed

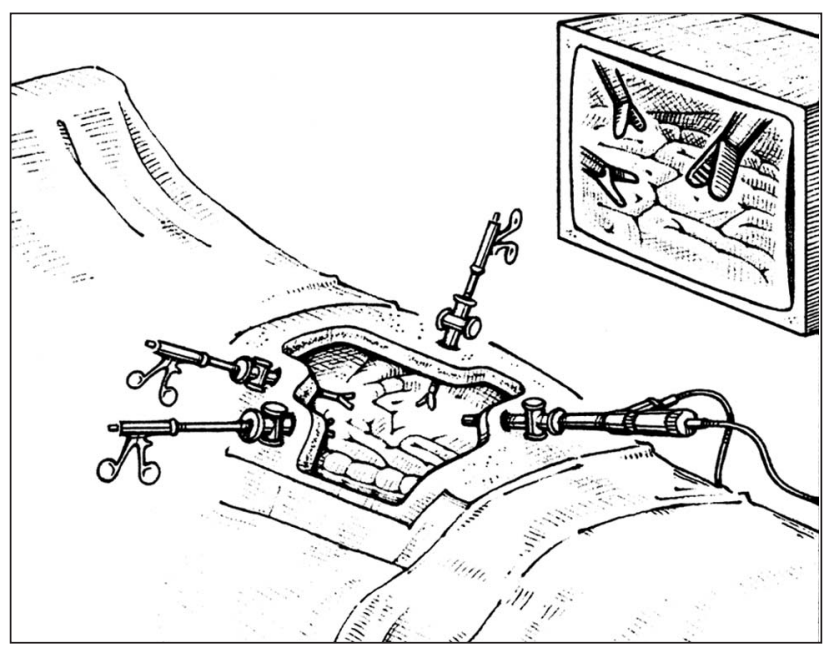

Fig. 1. A cutaway view of the abdomen with an endoscope (right) and three laparoscopic instruments.

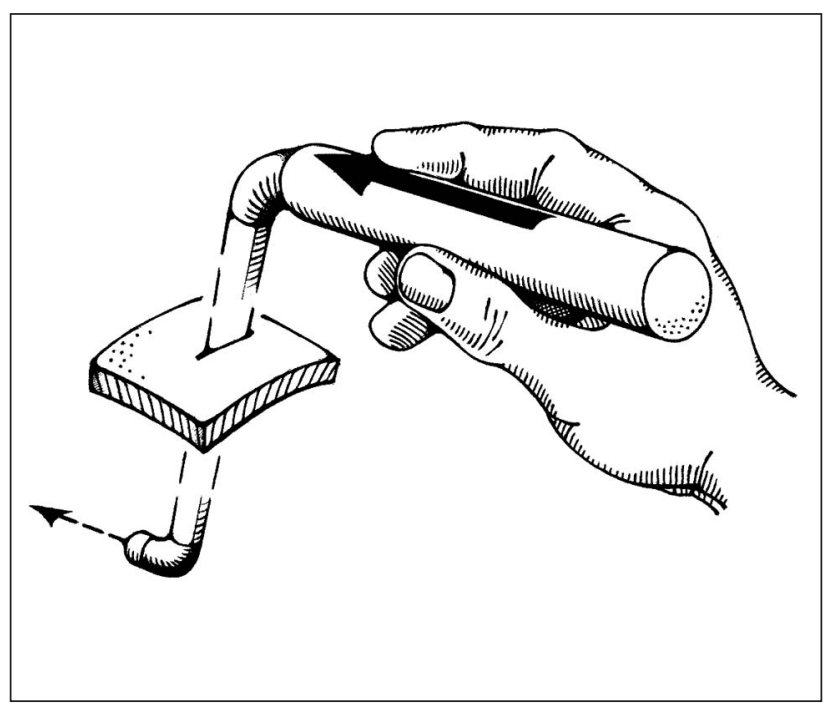

Fig. 3. The concept of the Endo-Periscope (reprinted from (15), (16) with permission of the ASME). from metal rings that are connected by hinges and controlled by cables (Figure 5) [5]-[7]. The rotation axes of the hinges are mutually rotated over $90^{\circ}$, creating a sequence of universal joints that are placed behind each other. The cables slide through tiny sleeves, which are soldered to the rings to keep the cables in position. The mechanism can be bent in all directions but cannot be compressed in axial direction.

In gastroscopes and colonoscopes, the mechanism is constructed from approximately 15 rings. Depending on the application, the diameter varies from 6-12 mm. At smaller diameters, the maneuverability is reduced to $1 \mathrm{DOF}$, making the mechanism simpler but restricting the surgeon's freedom of motion. The bending angle depends on the number and shape of the rings and usually differs for left/right and up/down tip rotations. At large bending angles up to $180^{\circ}$, the bending radius reaches a minimum value of approximately $10 \mathrm{~mm}$ at the inside bend. Disadvantages of the mechanism are that it is hard to realize a very small bending radius and almost impossible to miniaturize the diameter to a few

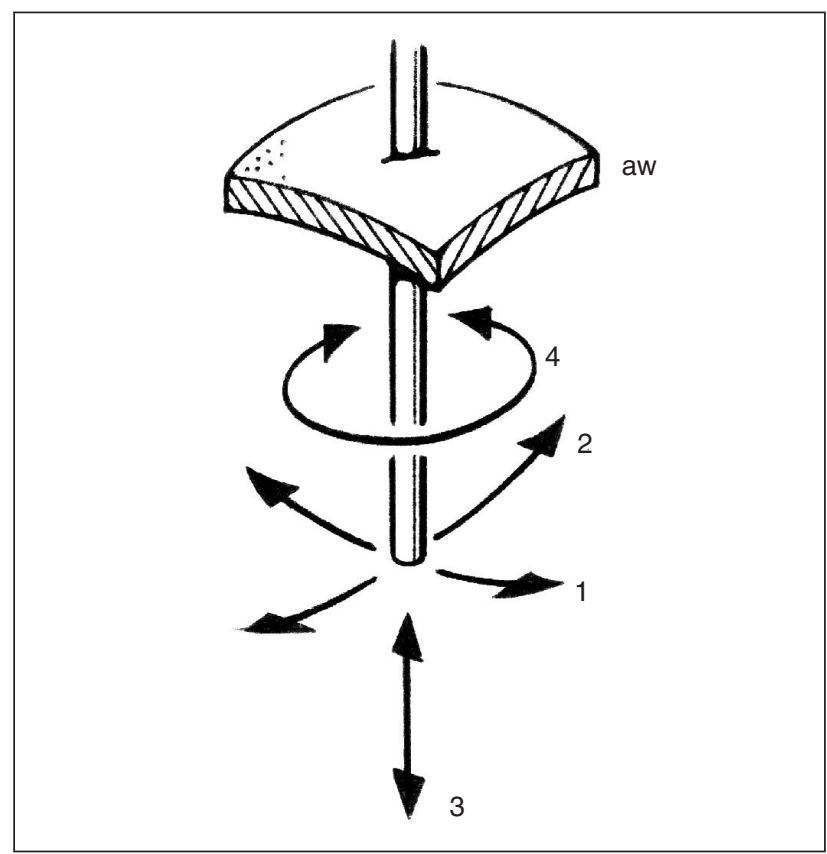

Fig. 2. The four degrees of freedom of a standard endoscope (aw $=$ the abdominal wall).

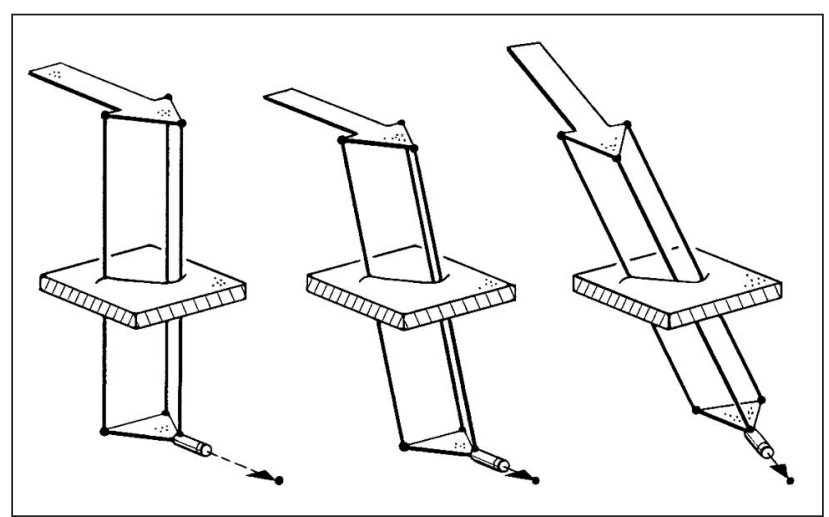

Fig. 4. Simplified line drawing showing the working principle of the spatial parallelogram-mechanism in the EndoPeriscope (reprinted from (15) with permission of the ASME). 
millimeters without restricting maneuverability or making the construction very fragile. The manufacturing requires a large amount of time-consuming manual assembling methods such as soldering and riveting, making the mechanism rather expensive. As a single unit, the mechanism is commercially available for a price of several hundred dollars (U.S.).

In an attempt to solve these problems, a number of alternative spine mechanisms have been developed [7], [8]. One of the simplest constructions is the mechanism in Figure 6, consisting of a flexible tube with a large number of notches that are mutually rotated over $90^{\circ}$ [9]-[11]. The tube contains four channels to keep the cables in position. Although it has a simpler construction, the disadvantages of this mechanism are that it is again hard to realize a very small bending radius, and it is

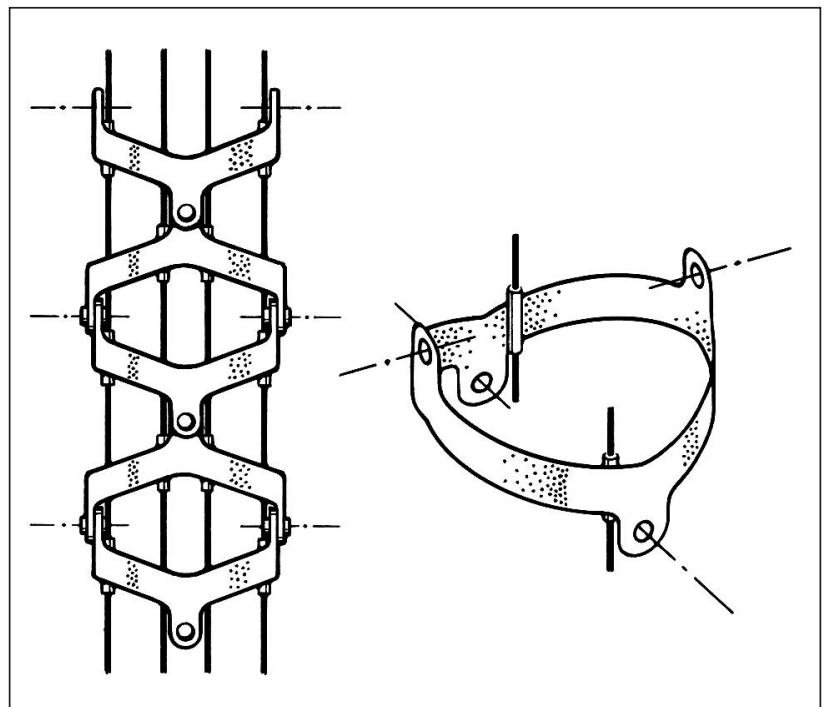

Fig. 5. A conventional steerable spine mechanism in colonoscopes and gastroscopes, consisting of a series of rings that are connected by hinges and controlled by cables (reprinted from (7) with permission of Mary Ann Liebert, Inc.). A close-up of one ring is shown at right.

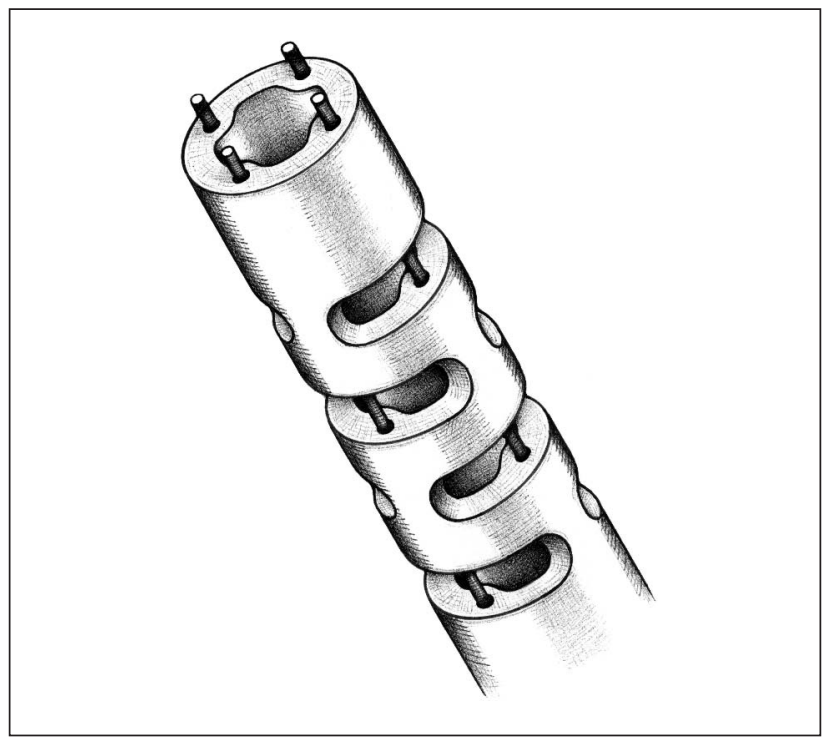

Fig. 6. An alternative steerable spine mechanism consisting of a flexible tube with notches. much stiffer than the mechanism with hinges. Manufacturing the channels and the notches is still quite labor intensive, and miniaturization makes the construction very fragile, with the risk of fatigue and fracture at the location of the notches. Since the mechanism is not applied in commercial endoscopes, no data have been found about the bending angle and radius.

\section{Working Principle of Tentacles of Squid}

Biological spine mechanisms can be found in the bodies and legs of arthropoda (such as insects) and vertebra (such as mammals). The spine serves as a flexible but incompressible support to which the muscles are attached. Cephalopods, such as squid, however, lack hard skeletal support and are still able to move their tentacles and arms in a large variety of poses. Figure 7 shows a schematic cross section of the tentacle of a squid [12], [13]. The tentacle is surrounded by longitudinal muscle layers (LML) and helical muscle layers (HML). The cross section contains a ring of longitudinal muscle bundles (LMB), which are enclosed by transverse muscle fibers (TMF) and circular muscle fibers (CMF).

The tentacle functions as a muscular hydrostat. Since the volume of the tentacle stays equal, its stiffness, curvature, and length can be changed by the interaction between the longitudinal retractor muscles (LML and LMB) and the circular and transversal extensor muscles (CMF and TMF). Contracting the retractors while relaxing the extensors will retract the tentacle and increase its diameter. Contracting the extensors while relaxing the retractors will extend the tentacle and decrease its diameter. Tensioning all muscles will stiffen the tentacle so it can exert forces on the environment. The helical muscle layers (HML) can exert a torque around the tentacle and may also help the tentacle to extend or retract, depending on their variable angle of pitch. The longitudinal muscle bundles (LMB) play a role not only in retracting the tentacle but also in bending it in a certain direction.

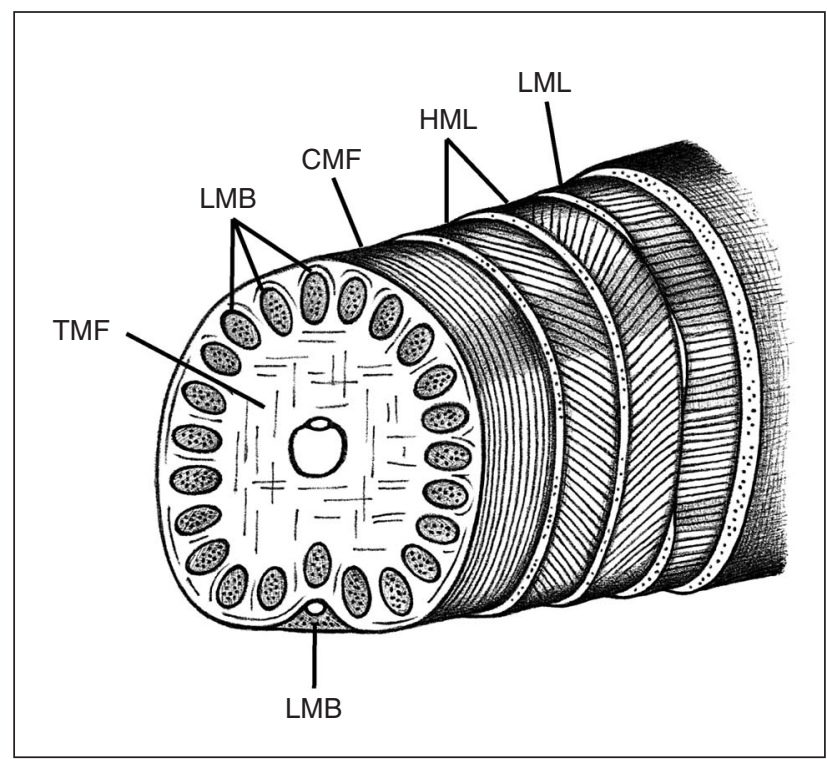

Fig. 7. A schematic cross section of the tentacle of the loliginid squid (redrawn from (13)). The tentacle is surrounded by longitudinal and helical muscle layers (LML and $\mathrm{HML}$ ). The cross section contains a ring of longitudinal muscle bundles (LMB), which are enclosed by transverse and circular muscle fibers (TMF and CMF). 
Its compressibility and adjustable stiffness give the tentacle a behavior comparable to a technical spring. The idea to replace the incompressible spine mechanism with a compressible spring leads to a new method to minimize bending radius and to simplify the construction of the Endo-Periscope.

\section{Compressible Steerable Spring Mechanisms}

In 1999-2000, a first, Ø15 mm prototype of the EndoPeriscope was developed in which the spine mechanism was replaced by a compression spring [14]-[16]. The spring is controlled by two cables; one is always completely tightened. If the other cable is released, the compression spring will push itself around with a very small radius until it reaches a maximum bending angle of $180^{\circ}$ with a minimum bending radius of $2.5 \mathrm{~mm}$ at the inside bend [see Figure 8(a)].

To keep the two cables in position, a new kind of spring, called the ring-spring was designed [Figure 8(b)]. It consists of a series of rings made from spring metal, and the rings are bent and welded to each other in pairs. The rings contain holes to keep the cables in position. The ringspring, for which a patent was applied for, combines very high torsion stiffness with low, asymmetric bending stiffness; it is easier to bend the spring in the plane through the holes than perpendicular to that plane. In the first prototype of the Endo-Periscope, the ring-spring was used only to control up/down tip rotations. Left/right tip rotations are still controlled by a hinge that connects the ring-spring to the endoscope shaft.

In 2001-2002, a second, Ø12 mm prototype with a simplified construction was developed [15], [16]. The hinge was removed and a new ring-spring with symmetric bending stiffness was designed to control both up/down and left/right tip rotations (Figure 9). The new ring-spring was constructed from a series of spring segments rotated over $90^{\circ}$ and connected to each other. The spring is controlled by four cables, which are released in such a way that the bending radius of the tip is minimized for all bending directions. The tip can be steered up/down and left/right until it reaches a maximum bending angle of $125^{\circ}$ with a minimum bending radius of $6 \mathrm{~mm}$ at the inside bend.

A series of extensive tests with the two prototypes has shown the ring-spring works very well, offering a simple and reliable alternative for the spine mechanism. The compressibility leads to a strong reduction in bending radius and the spring-metal rings have a simple 2-D shape, making them easy to manufacture at very low costs. A disadvantage, however, is that it is difficult to miniaturize the ring-spring to a diameter of $5 \mathrm{~mm}$ or less while keeping production costs very low. In the two prototypes, the spring-metal rings have been manually connected using spot welds, a manufacturing method that turned out to be rather labor intensive. Automatic spot welding or laser welding at miniature scale requires precise and expensive equipment and seems not very suitable for low-cost mass production.

\section{Minimizing Diameter by Maximizing Number of Cables}

The ring-spring is controlled by four cables, which are kept in position by the holes. The construction is equivalent to the universal joints in the spine mechanism of Figure 5, with the difference that the hinges are replaced by leaf springs. Although the ring-spring offers a simple solution to combine pushing with guiding cables, a construction with standard coil springs would be preferable. Coil springs are commercially available in an enormous range of sizes and can be obtained at very low costs.

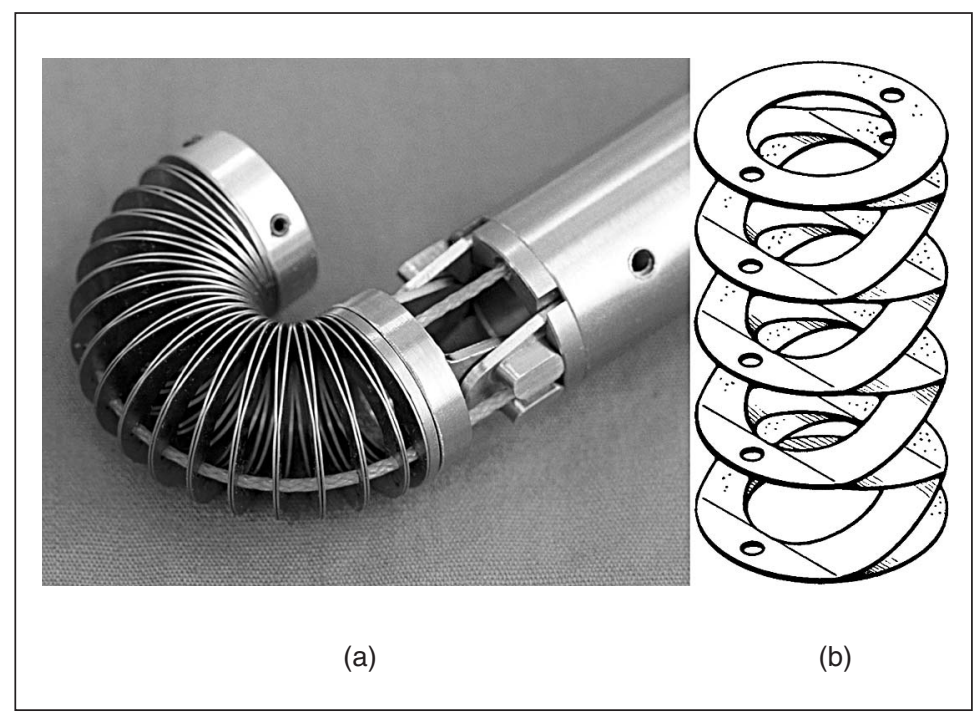

Fig. 8. Steerable tip (a) and ring-spring (b) of the Endo-Periscope I (reprinted from (14), (15), (16) with permission of Taylor \& Francis and the ASME). The tip can be steered up/down between $0^{\circ}$ and $180^{\circ}$ and left/right between $-60^{\circ}$ and $+60^{\circ}$. In the figure, the tip is bent over $180^{\circ}$.

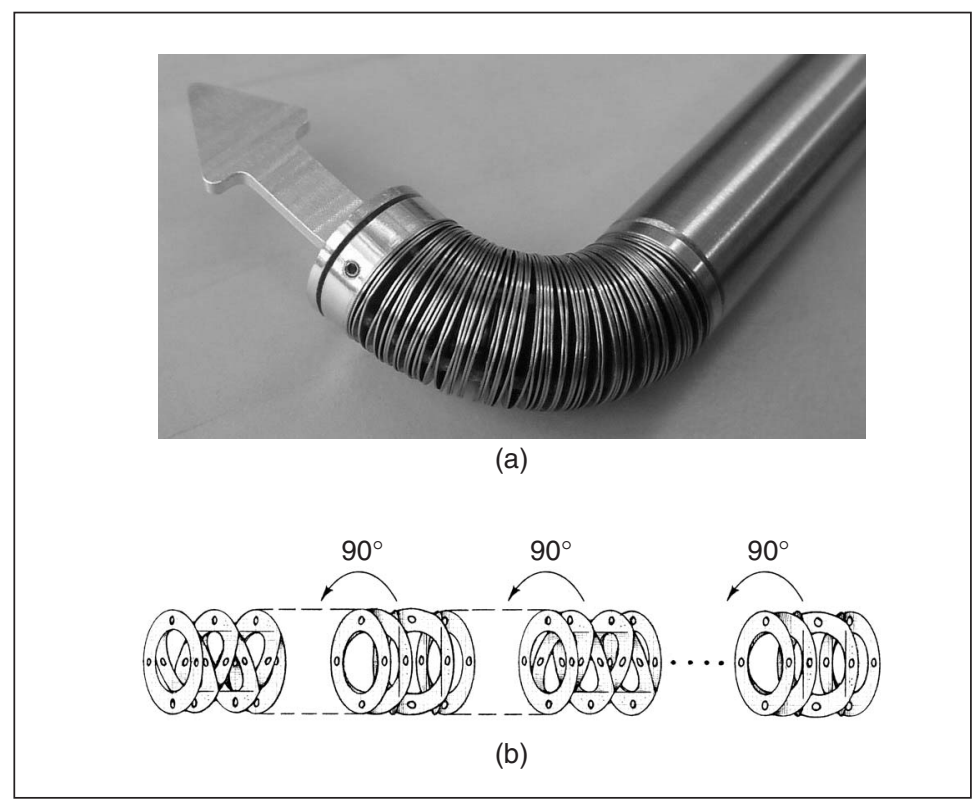

Fig. 9. The steerable tip (top) and ring-spring (bottom) of the EndoPeriscope II (reprinted from (15), (16) with permission of the ASME). The arrow visualizes the camera's line of sight and the tip can be steered up/down and left/right between $-125^{\circ}$ and $+125^{\circ}$. 
Radial cable displacements can be eliminated if the ringspring is replaced by two coil springs: an inner spring and an outer spring with a larger diameter than the inner spring. Placing the inner spring in the outer spring results in a ringshaped slot through which the cables can be guided. If the thickness of the slot equals the thickness of a cable, then the cables will be fixed in radial position. Tangential displacements can still occur because of the tangential distance between the cables.

Controlling left/right and up/down tip rotations is in principle possible with a minimum amount of three cables. In conventional spine mechanisms and in the ring-spring, four cables are used since this is easier to combine with the universal joint construction. Biological mechanisms, however, are often strongly redundant to make them more robust against failure. A tentacle contains a ring of longitudinal muscle bundles, enabling the squid to still use its tentacle when one or more bundles are not functioning well.

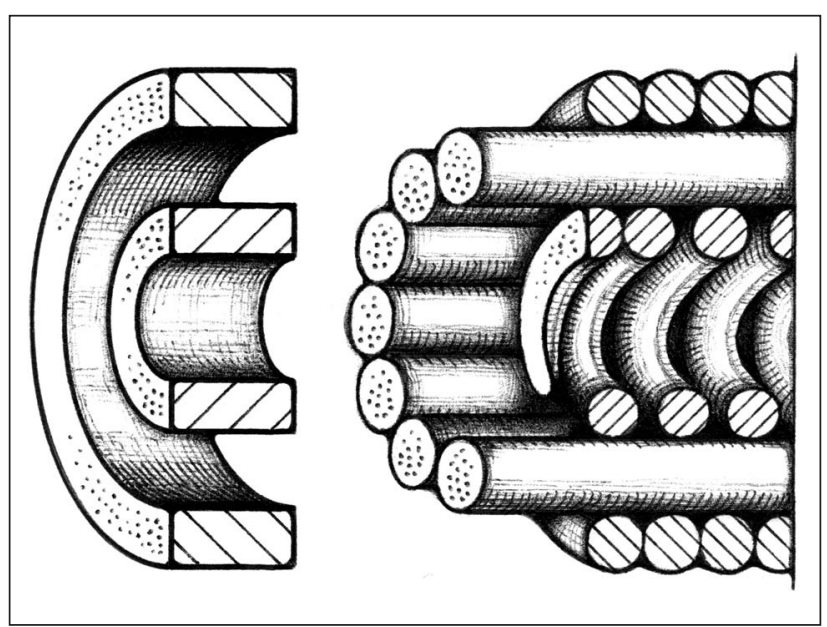

Fig. 10. A cross section of the cable-ring mechanism, showing the inner spring, the outer spring, and the ring of cables. The cables are fixed to the endoscope tip by squeezing them between two rings.

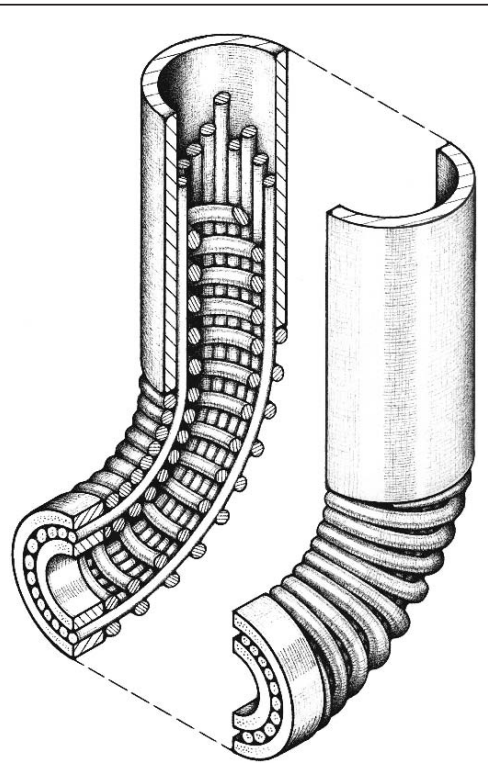

Fig. 11. A cross section of the tip of the Endo-Periscope III.
Using this idea, the number of cables between the two springs can be enlarged until the ring-shaped slot is completely filled. The distance between the cables is then zero, so the cables are fixed in tangential position as well. This results in a ring of cables fitting precisely between the two coil springs (Figure 10). The cable ring can be fixed to the endoscope tip by squeezing the cables between two rings of which the difference in radius is slightly smaller than the thickness of a cable. This method was tested in practice and proved a strong and reliable fixation method. The resulting cable-ring mechanism combines pushing with guiding while keeping the cables in position. Sliding the cables with respect to each other will bend the tip in a required direction.

\section{Parallelogram-Control of the Endo-Periscope}

In 2003-2004, the cable-ring mechanism was used to develop a third prototype of the Endo-Periscope. The steerable tip is controlled by a new spatial parallelogram-mechanism of which the working principle is visualized in Figures 11 and 12. The cables are fixed between two rings at the tip and guided through shaft and handgrip where they are fixed between two other rings. These rings form a carriage that can slide in the handgrip. The cable ring contains an inner spring along the entire length of the cables and is surrounded by three outer springs: a tip spring between tip and shaft, a handgrip spring between shaft and handgrip, and a compensation spring in the handgrip. The four springs keep the cables in position and prevent them from buckling. The handgrip spring is designed as a closed tension spring to increase stiffness of the connection between the handgrip and shaft. The other three springs are compression springs, with the compensation spring stronger than the tip spring.

The interaction between the tip spring and the compensation spring is used to obtain parallelogram control. The compensation spring pushes the carriage and the cable ring to the right. The resulting tension force on the cables is so strong that the tip spring is completely compressed in the straight

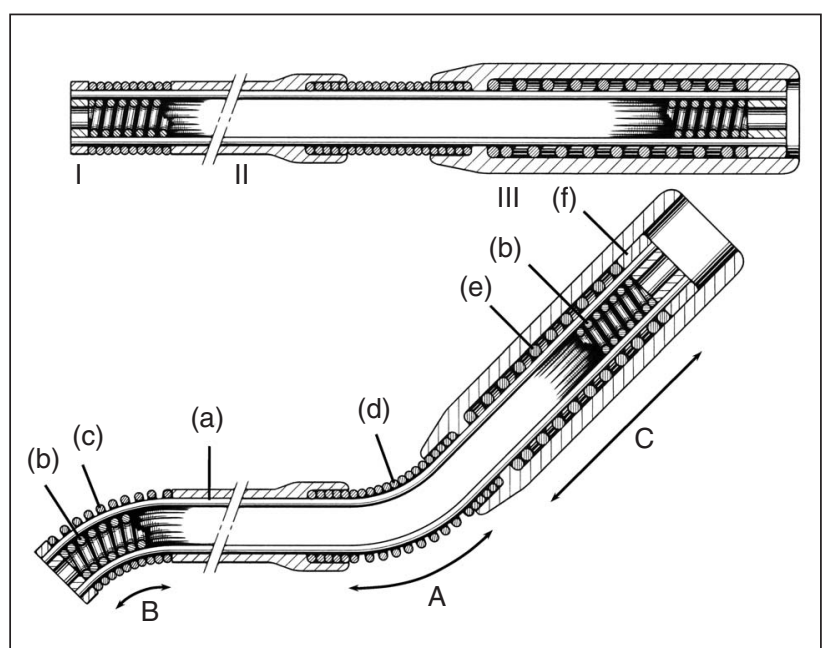

Fig. 12. A cross section of the parallelogram-mechanism of the Endo-Periscope III in the straight (top) and bent position (bottom), showing the tip I, shaft II and handgrip III, with cable ring (a), inner spring (b), tip spring (c), handgrip spring (d), compensation spring (e), and carriage (f). A, B, and C are parts of the cable at the bottom. From the cable ring and the inner spring, only the beginning and end are shown. 
position (Figure 12, top). When the handgrip is bent (Figure 12, bottom), Part A of the cable at the outside of the curvature becomes longer. Part B of that cable, however, cannot be shortened since the tip spring is completely compressed. Instead, Part $\mathrm{C}$ becomes shorter and the carriage moves downward. As a result, the other cables are released. These cables slide through the handgrip and shaft and cause the tip spring to bend with a very small radius until it reaches the same angle as the handgrip.

\section{Prototype of the Endo-Periscope}

Figure 13 shows the resulting prototype in straight and bent positions. The endoscope is completely hollow with inner and outer diameters of 2 and $5 \mathrm{~mm}$, respectively. This makes the endoscope more than two times as thin as the second prototype. The cable ring consists of 22 steel cables with a thickness of $0.45 \mathrm{~mm}$ (Figure 14). The ring-shaped slot between the inner and outer springs is $0.50 \mathrm{~mm}$ thick, creating $0.05 \mathrm{~mm}$ in clearance for the free motion of the cables.

Since it was not completely clear beforehand how the mechanism would behave at large tip rotations, the endoscope was equipped with three adjustment mechanisms to fine-tune the spring force of the compensation spring and the length of the tip and handgrip springs. The adjustment-mechanisms can be omitted in future prototypes, leaving a cheap construction of cables, coil springs, rings, and tubes.

Figure 15 shows a close-up of the steerable tip. The mechanism is completely symmetric and the tip can be bent in all directions up to a maximum bending angle of $110^{\circ}$ with a minimum bending radius of $8 \mathrm{~mm}$ at the inside bend. When the handgrip is bent over a larger angle, the friction and the changing balance of spring forces cause the cables to buckle within the $0.05-\mathrm{mm}$ clearance for free motion. This restricts a further rotation of the tip, distorts the parallelogram behavior, and does not allow the bending radius to become smaller. Although a $110^{\circ}$ bending angle seems enough for laparoscopic applications, the mechanism is currently being further improved into a new $\emptyset 1.5 \mathrm{~mm}$ prototype in which the tip can bend $180^{\circ}$ in all directions with a bending radius of 5 $\mathrm{mm}$ at the most. This prototype is intended for microsurgical applications - such as eye, spinal cord, or brain surgery - in which the available space is strongly restricted and surrounded by vulnerable tissues that are easily damaged.

\section{Discussion}

Table 1 shows the difference between the 2-DOF steerable tips of conventional gastroscopes and colonoscopes and the prototypes of the Endo-Periscope in terms of diameter, maximum bending angle, and minimum bending radius. The table shows that the compressible spring mechanisms lead to a decrease in bending radius and the cable-ring mechanism can be constructed with a much smaller diameter than conventional spine mechanisms, without restricting maneuverability of the steerable tip. It must, of course, be noted that the bending angle and radius depend on a number of design parameters, such as the length of the steerable tip, the shape of the rings, the stiffness of the springs, the stiffness of the cabling guided through the endoscope lumen, and, if present, the stiffness of the sheathing around the steerable tip. Therefore, the table can only be used as an indication and not as an absolute measure. Since the third prototype has a novel construction for which no examples had existed, the mechanical construction is not yet optimized. The prototype has a number of imperfections, resulting in a relatively large bending radius. It is expected that this disadvantage will be solved in the new $\emptyset 1.5 \mathrm{~mm}$ prototype.

The cable-ring mechanism is much simpler than conventional spine mechanisms, consisting only of standard parts such as cables, coil springs, rings, and tubes. These parts are commercially available in very large quantities and can be obtained at very low costs. Special machining is hardly needed and the manufacturing contains no time-consuming assembling methods such as soldering or riveting. Since the mechanism can be manufactured at very low costs, it is expected that the endoscope can be sold as a disposable device, using the cheap, highquality miniature cameras that are currently being developed

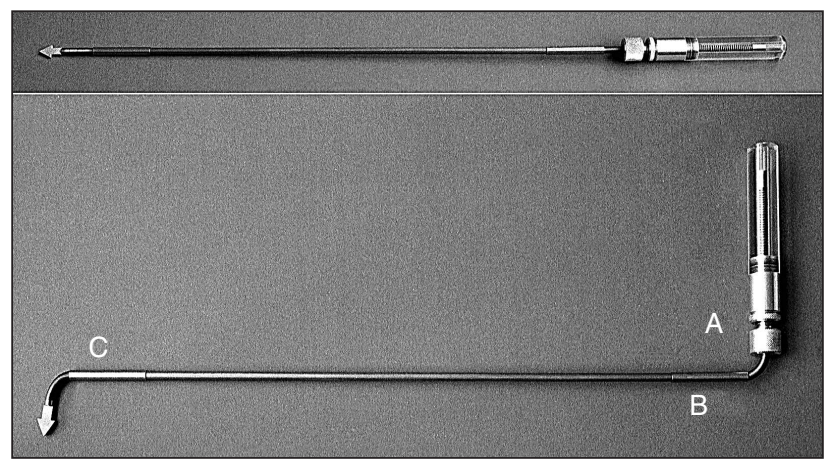

Fig. 13. The Endo-Periscope III in straight and bent position. The prototype contains three adjustment-mechanisms-A, B, and $\mathrm{C}$-to fine-tune the spring force of the compensation spring and the length of the tip and handgrip springs.

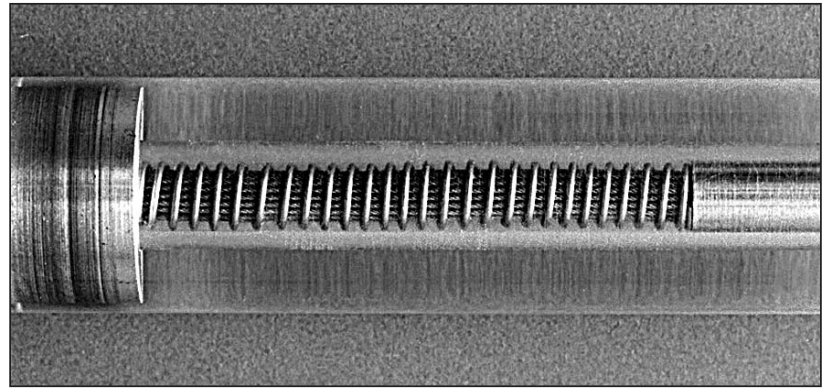

Fig. 14. A close-up of the transparent handgrip showing the cable-ring mechanism.

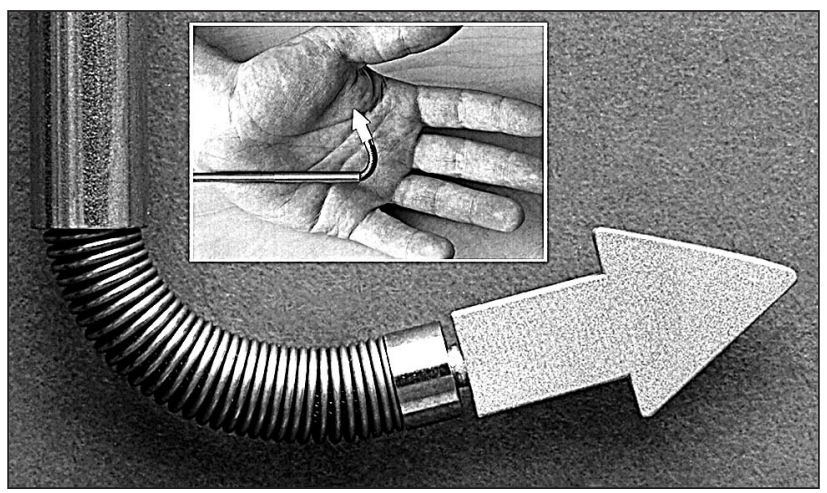

Fig. 15. A close-up of the steerable tip of Endo-Periscope III. The arrow visualizes the camera's line of sight and the tip can be steered in all directions between $-110^{\circ}$ and $+110^{\circ}$. 
for mobile phones and digital photography. Sterilization after the operation is then not needed, making it unnecessary to sheath the steerable tip or to provide arrangements for disassembly, which would make the mechanism more complicated. It is expected that the cablering mechanism can be produced in large series and sold for approximately US\$20 per unit, which is much cheaper than the price for the conventional spine mechanism in Figure 5.

Several tests with the second prototype have shown that an inexperienced person needs approximately 15-20 min of training time to get experienced in controlling the EndoPeriscope. The parallelogram-method of controlling is thus quite easy to learn, making the Endo-Periscope very interesting for surgical practice. Although the third prototype has a diameter of $5 \mathrm{~mm}$, it seems easy to miniaturize the cable-ring mechanism further to a diameter of $1.5 \mathrm{~mm}$ or even less by using standard $\emptyset 0.2 \mathrm{~mm}$ steel cable and $\varnothing 0.2 \mathrm{~mm}$ spring steel wire. This makes it very useful for a number of other applications, such as miniature steerable instruments and catheters.

A disadvantage of the cable-ring mechanism as compared to spine mechanisms or the ring-spring is that its torsionstiffness is lower. This is not a problem for steerable endoscopes, which, in principle, do not contact the tissue. It can, however, be a drawback when applied in a steerable instrument used for manipulating the tissue. The torsion-stiffness increases strongly if the outer spring is surrounded by a flexible, torsion-stiff sleeve that is braided from very thin helical steel cables, equivalent to the helical muscle layers in squid tentacles. Torsion-stiff sleeves are already used in gastroscopes and colonoscopes and are commercially available at very low costs. Therefore, it seems this problem is not difficult to overcome.

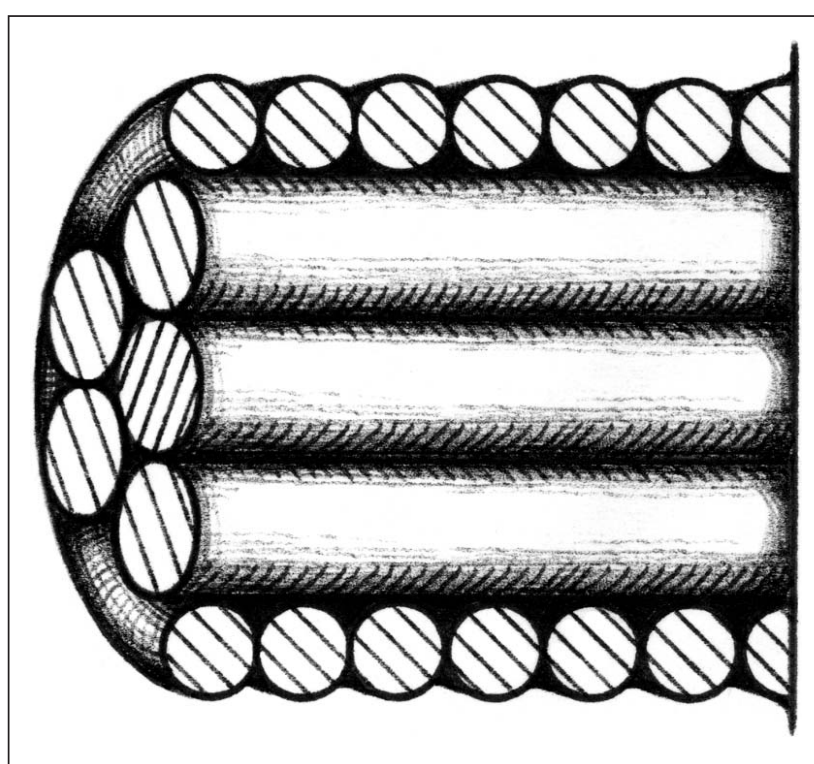

Fig. 16. The cable-ring mechanism with a central cable for use in a steerable instrument.
When the cable-ring mechanism is applied in a steerable instrument (see Figure 16), the inner spring can be replaced by a cable that can be moved forward and backward to drive a grasper or a pair of scissors connected to the instrument tip. The cable ring can be used to steer the tip in all directions, while the outer spring keeps the cables together. Using standard $\varnothing 0.2 \mathrm{~mm}$ steel cable and $\varnothing 0.2 \mathrm{~mm}$ spring steel wire, it seems possible to miniaturize this mechanism to a diameter of $\varnothing 1.0 \mathrm{~mm}$, making it very suitable for steerable microinstruments or catheters. Conventional 2-DOF steerable instruments usually contain a spine mechanism of miniature hinges and pulleys (Figure 17) [7], [8], [17]. The complex shape of these elements makes it very difficult to miniaturize these instruments at low costs. This makes the cable-ring mechanism a promising alternative.

Although the cable-ring mechanism was inspired by mechanisms in nature, the working principle differs from the

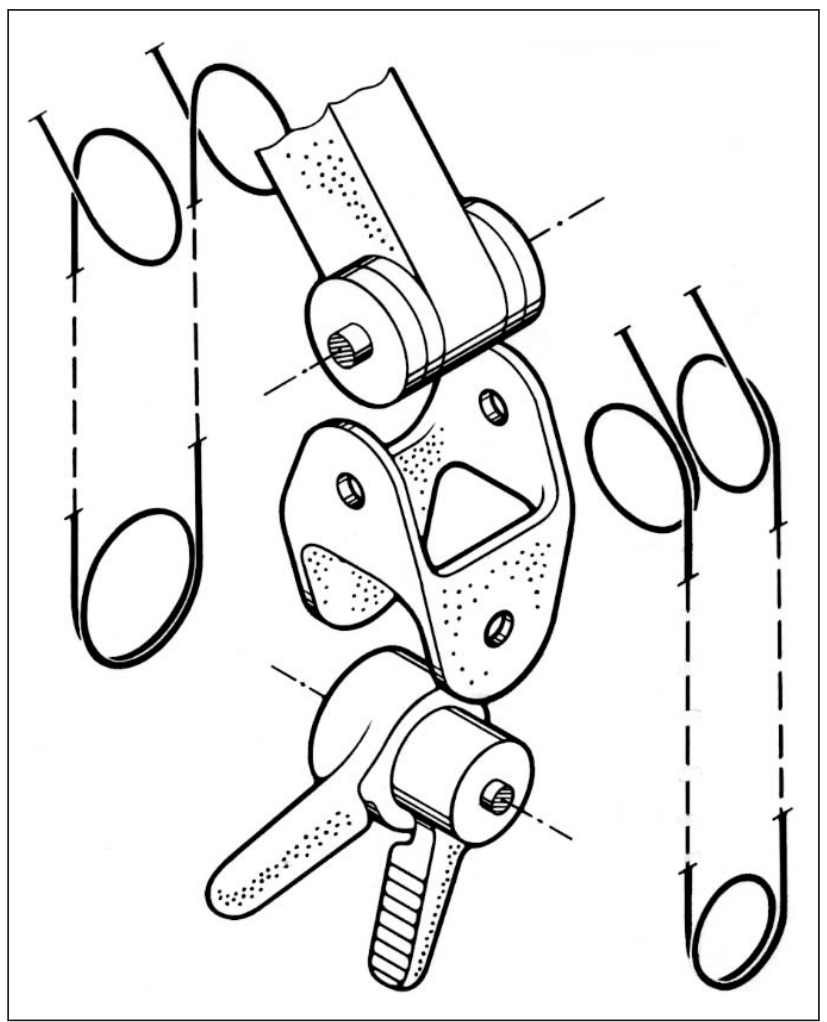

Fig. 17. The tip of a steerable instrument for medical applications (reprinted from (7) with permission of Mary Ann Liebert Inc.). The mechanism is ingenious but difficult to miniaturize (at low cost) to a diameter of a few millimeter. 
tentacles of squid. The ring of muscle bundles in the tentacle has a redundant function, whereas the function of the ring of cables is to avoid tangential cable displacements. Another difference is that the tentacle does not contain compression springs and it can be twisted in an axial direction as well. The goal of the research, however, is not to imitate biological mechanisms but to use them as inspiration and to take the best of both the technical and biological worlds for the development of innovative technology.

An application for a patent for the cable-ring mechanism has been submitted, covering a large number of medical and industrial applications. The mechanism is currently being commercialized, and it is the authors' wish that this will lead to a range of new, useful instruments for laparoscopic surgery.

\section{Acknowledgments}

The research of Paul Breedveld has been made possible by a fellowship of the Royal Netherlands Academy of Arts and Sciences. The first two prototypes have been developed in close cooperation with the Hirose \& Yoneda Laboratory of the Tokyo Institute of Technology. We would like to thank our designer Ad v/d Geest for manufacturing the third prototype of the Endo-Periscope.

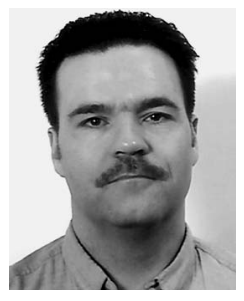

Paul Breedveld studied mechanical engineering at the Delft University of Technology, the Netherlands, where he received his M.Sc. degree in 1991 and his Ph.D. degree in 1996, both with honors. His $\mathrm{Ph} . \mathrm{D}$. study was focused on the development of a man-machine interface for a space manipulator. He worked as a postdoctoral fellow from 1997-2001at the Delft University of Technology and at the Tokyo Institute of Technology on the development of steerable endoscopes for minimally invasive surgery. He received a number of awards and is currently doing research on steerable surgical instruments and intestine intervention devices, financed by a fellowship from the Royal Netherlands Academy of Arts and Sciences.

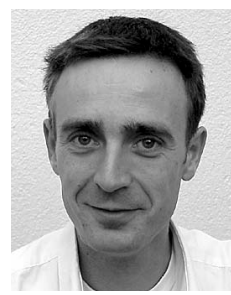

Jules S. Scheltes studied mechanical engineering at the Delft University of Technology, the Netherlands, where he received his M.Sc. degree in 1996 and his Ph.D. degree in 2003. His Ph.D. study was focused on the development of mechanical couplings for connecting blood vessels during minimally invasive bypass procedures on the heart. After his Ph.D. study, he started working on the development of steerable endoscopes for minimally invasive surgery. In 2004, he founded the company DEAM-engineering that focuses on the development of steerable surgical instruments.

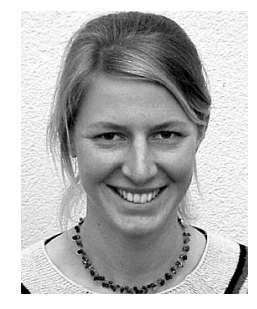

Esther M. Blom studied human kinetic technology at the Hague University in the Netherlands and received her bachelor's degree with honors in 2002. In 2002-2003, she worked at the Delft University of Technology on the development of steerable endoscopes and on setting up a laboratory for experiments on animal tissue. In 2003-2004 she went to India to educate local technicians and to manufacture special aids for handicapped children. Currently, she is doing research at the Delft University of Technology on registration of information processes in operating rooms.

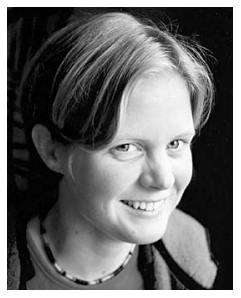

Johanna E.I. Verheij studied human kinetic technology at the Hague University in the Netherlands and received her bachelor's degree with honors in 2002. In 2002-2003, she worked at the Delft University of Technology on the development of steerable endoscopes and on setting up a laboratory for experiments on animal tissue. In 2003-2004, she went to India to educate local technicians and to manufacture special aids for handicapped children. Currently, she is working as a technical advisor at Aquarius, a Dutch company specializing in fabricating ortheses.

Address for Correspondence: Paul Breedveld, Delft University of Technology, Faculty of Mechanical Engineering, Man-Machine Systems Group, Mekelweg 2, 2628 CD Delft, The Netherlands. Phone: +31 152785232. Fax: +31 15 2784717. E-mail: p.breedveld@wbmt.tudelft.nl.

\section{References}

[1] G.J.F. Smets, C.J. Overbeeke, and M.H. Stratmann, "Depth on a flat screen," Perceptual and Motor Skills, vol. 64, pp. 1023-1034, 1987.

[2] G.J.F. Smets and C.J. Overbeeke, "Trade-off between resolution and interactivity in spatial task performance," IEEE Comput. Graph. Appl., pp. 46-51, Sept. 1995.

[3] W. Pasman, G.J.F. Smets, and P.J. Stappers, "Effects of image quality, number of selectable viewpoints, and way to select the viewpoints in X-ray luggage inspection," Presence, vol. 6, no. 3, pp. 268-281, 1997.

[4] F. Voorhorst, D.W. Meijer, C.J. Overbeeke, and G.J.F. Smets, "Depth perception in laparoscopy through perception-action coupling," Minimally Invasive Therapy and Allied Technols., vol. 7, no. 4, pp. 325-334, 1998.

[5] M.O. Schurr, A. Melzer, P. Dautzenberg, B. Neisius, R. Trapp, and G. Buess, "Development of steerable instruments for minimal invasive surgery in modular conception," Acta Chirurgica Belgica, vol. 93, pp. 73-77, 1993.

[6] A. Melzer, M.O. Schurr, M.M. Lirici, B. Klemm, D. Stöckel, and G. Buess, "Future trends in endoscopic suturing," Endoscopic Surgery, vol. 2, pp. 78-82, 1994.

[7] P. Breedveld, H.G. Stassen, D.W. Meijer, and J.J. Jakimowicz, "Manipulation in laparoscopic surgery-overview of impeding effects and supporting aids," $J$. Laparoendoscopic Advanced Surgical Techniques, vol. 9, no. 6, pp. 469-480, 1999.

[8] F. Cepolina and R.C. Michelini, "Review of robotic fixtures for minimally invasive surgery," Int. J. Med. Robot. Comput. Assisted Surgery, vol. 1, no. 1, pp. 43-63, 2004

[9] E. L. Adair, "Steerable sheath for use with selected removable optical catheter," U.S. Patent 5325 845, July 5, 1994.

[10] A. DeLaRama, L.A. Dial, R.J. Harp, T.L. Hursman, A.S. Soria, R.J. Serra, and S. Tarpening, "Bi-directional and multi-directional miniscopes," U.S. Patent 5 381782 , Jan. 17, 1995.

[11] H.E. Harder, H.I. Jensen, and A.W. Speitling, "An elongate element for transmitting forces," European patent application EP 0889252 A2, Jan. 7, 1999.

[12] W.M. Kier, "The functional morphology of the musculature of squid (Loliginidae) arms and tentacles," J. Morphol., vol. 172, pp. 179-192, 1982.

[13] J.L. van Leeuwen and W.M. Kier, "Functional design of tentacles in squid: Linking sarcomere ultrastructure to gross morphological dynamics," Philosophical Trans. Royal Soc., Ser B, Biological Sci., vol. 352, pp. 551-571, 1997.

[14] P. Breedveld and S. Hirose, "Development of the Endo-Periscope," Minimally Invasive Therapy and Allied Technols., vol. 10, no. 6, pp. 315-322, 2001.

[15] P. Breedveld and S. Hirose, "Development of steerable endoscopes to improve depth perception during laparoscopic surgery," in Proc. DETC'02 ASME Design Eng. Conf., 2002, pp. 1-6.

[16] P. Breedveld and S. Hirose, "Design of steerable endoscopes to improve the visual perception of depth during laparoscopic surgery," ASME J. Mech. Design, vol. 126, pp. 2-5, Mar. 2004.

[17] A.J. Madhani and J.K. Salisbury, "Wrist mechanism for surgical instrument for performing minimally invasive surgery with enhanced dexterity and sensitivity," U.S. Patent 5797 900, p. 1-16, Aug. 25, 1998. 